\title{
A New Method for Internal Force Detection of Steel Bars Covered by Concrete Based on the Metal Magnetic Memory Effect
}

\author{
Caoyuan Pang ${ }^{1}{ }^{\mathbb{D}}$, Jianting Zhou ${ }^{1, * \mathbb{C}}$, Qingyuan Zhao ${ }^{1}$, Ruiqiang Zhao ${ }^{2}$, Zhuo Chen ${ }^{1}$ and \\ Yi Zhou ${ }^{3}$ \\ 1 College of Civil Engineering, Chongqing Jiaotong University, Chongqing 400074, China; \\ 622160086030@mails.cqjtu.edu.cn (C.P.); 622170970111@mails.cqjtu.edu.cn (Q.Z.); \\ zhuochen@cqjtu.edu.cn (Z.C.) \\ 2 Engineering Research Center of Bridge Structure and Material in the Mountainous Area of Ministry of \\ Education and College of Materials Science and Engineering, Chongqing Jiaotong University, Chongqing \\ 400074, China; rqzhao@cqjtu.edu.cn \\ 3 Chongqing Yapai Bridge Engineering Quality Inspection Co., Ltd., Chongqing 401120, China; \\ boatzy@163.com \\ * Correspondence: jtzhou@cqjtu.edu.cn; Tel.: +86-23-6265-2702
}

Received: 13 May 2019; Accepted: 4 June 2019; Published: 6 June 2019

\begin{abstract}
In this paper, the specimens of steel bars covered by concrete (SBCC) are taken as research objects, and a new method for steel bar internal force detection based on the metal magnetic memory effect is proposed. The variation law of the self-magnetic flux leakage (SMFL) signals on the surfaces of SBCC specimens with loading tension and the variation of the SMFL signals along the axial positions of specimens under different tensile forces are studied. The results show that when the loading tension is about $90 \%$ of the yield tension, the tangential component of the SMFL signal has a maximum extreme point. The distribution of the SMFL signals along the axial position shows a smooth curve, where the values at both ends are small while the intermediate values are large. This paper also proposes the use of the "area ratio deviation parameter" to quantitatively calculate the internal forces of the steel bars. This parameter shows a significant linear relationship with the loading tension during the strengthening stage of the specimens. This method can supplement the existing steel bar stress detection methods and has prospective research value.
\end{abstract}

Keywords: metal magnetic memory; steel bar covered by concrete; stress nondestructive testing; strengthening stage

\section{Introduction}

There is a similar coefficient of linear expansion between steel bars and concrete, and the bonding performance between them is good. On the other hand, the alkaline environment of concrete can prevent the corrosion of steel bars to a certain extent. Therefore, the application of reinforced concrete in building structures is common [1,2]. With the development and popularization of prestressed reinforced concrete technology, the proportion of reinforced concrete bridges exceeds half of the total in bridge engineering around the world [3]. In reinforced concrete bridge structures, the stress of steel bars has always been a key indicator in bridge health and safety monitoring [4]. To determine how to carry out non-destructive detection of the stress of steel bars inside the concrete in a more accurate and convenient way, scholars all over the world have carried out a lot of researches.

The detection of steel bar stress in concrete structures is significant, and its detection methods are numerous. From the damage degree to the structure, the detection methods can be divided into 
damage detection (destruction, semi-destruction) and non-destructive detection [5,6]. Compared with damage detection, non-destructive detection has the advantages of high precision, small error, and no destruction to the structure, so it is used more now [7]. The traditional non-destructive stress detection methods, such as ultrasonic testing [8,9], eddy current testing [10,11], and X-ray testing [12,13], cannot detect early damage of steel bars [14]. In order to accurately detect the early damage of metal materials by means of non-destructive detection, some effective and new methods based on the physics have come into being [15-17]. Metal magnetic memory (MMM) detection technology is one of them [18,19], which was first proposed by Russian scholar Dubov [20]. The basic principle of MMM detection can be summarized as follows: Ferromagnetics indicate that magnetism is a basic property of matter, and that any substance is magnetic. When putting any substance in a magnetic field, under the action of a magnetic field, the substance will be magnetized. The reason why a substance is magnetic is because there is a phenomenon in which electrons rotate and spin around the nucleus, and the magnetic properties of the substance can be quantified by magnetic moments [21,22]. The steel bars in reinforced concrete structures are typical metal materials, and their interior structure can be regarded as being composed of many magnetic domains. The interface between the magnetic domains is called the magnetic domain wall [23]. When subjected to the geomagnetic field, the steel bars are magnetized to excite the magnetic fields around themselves, which form the initial magnetic fields under the action of the geomagnetic field. When the steel bars are subjected to the external tension, under the action of the tensile forces and the geomagnetic field, the magnetic domain structures orient and irreversibly reorient, with the magnetostrictive properties changing in the tensioned and deformed regions. In these regions, a fixed node of the magnetic domain emerges, and a magnetic pole is generated to form a demagnetizing field, so that the magnetic permeability of the stress-receiving areas of the steel bars is minimized, forming leakage magnetic fields on the surfaces as a result. The irreversible variation of this magnetic state is retained after the external tension is removed, so this phenomenon is called "metal magnetic memory" [24]. The leakage magnetic fields on the surfaces of the metal stress concentration zones have a maximum value of the tangential component $H_{\mathrm{p}}(x)$, and the normal component $H_{\mathrm{p}}(y)$ changes the sign and has a zero point, as shown in Figure 1, which is the commonly used criteria basis of the MMM method [25-27].

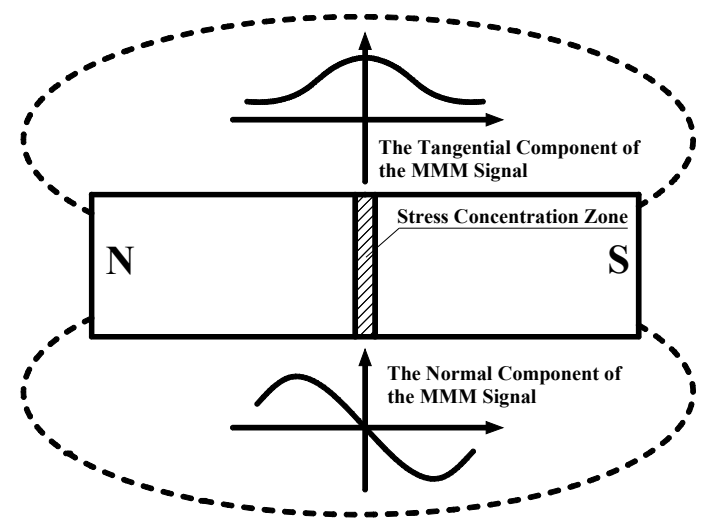

Figure 1. Schematic diagram of the commonly used criteria for stress concentration by the metal magnetic memory (MMM) method.

Compared with other non-destructive testing methods, the MMM method has unique advantages in the stress detection of metal materials. The MMM method is a magnetic non-destructive testing method that is more convenient compared to other magnetic non-destructive testing methods (such as the magnetic flux leakage detection method [28,29], the magnetic acoustic emission method [30], and the magnetic Barkhausen method [31]). It is a passive magnetic measurement method that does not require the use of an artificial magnetic field to excite the material, but instead uses the geomagnetic field as an excitation source. For the detection of defects and damage, it is only necessary to collect the self-magnetic flux leakage (SMFL) signal on the surface of the test piece by magnetic sensors under 
the combined action of the geomagnetic field and stress [32]. The MMM method is far more sensitive than other non-destructive testing methods in the detection of stress. It is extremely advantageous in the early damage detection of metal materials. Therefore, this technology has been widely used in the damage detection of metal pipes, metal containers, and turbine blades [33]. In addition, when the MMM method is used for detection, it is not necessary to perform special pre-cleaning on the surface of the specimen to be inspected, and the detection time of the process can be shortened. During the whole detection process, the magnetic sensor is not in contact with the test piece, and is suitable for the stress detection of steel bars with a protective layer of concrete [34,35]. Furthermore, the equipment used for detecting stress by the MMM method is simple and light, easy in operation, high in computer-automatic acquisition and control degree, and fast in detection speed, so it is suitable for large-area detection [34,36].

Researchers around the world have done a lot of researches on stress detection using the MMM method, and have achieved corresponding results. Dong [37] used 18CrNi4A-plate steel specimens to conduct tension-tension fatigue experiments, defined the stress concentration factor $K_{\mathrm{h}}$, and proposed a new method for calculating the stress concentration factor by using $H_{\mathrm{p}}(x)$. Wang [38] presented a linear magnetic charge model to predict the SMFL signals in a defect zone caused by local stress concentration, and observed through the tests that defects on the surface of a structure have much more effect on SMFL amplitude than those in the inside of the structure. Chen [39] used the Q235 steel to carry out a series of static tensile tests and conducted the finite element analysis, and concluded that the gradient of the normal component of SMFL signals determines the strain energy density built in the structure during the loading. Yu [40] studied the MMM signal of $20 \mathrm{~g}$ steel specimens during the static tensile loading process, and found that the MMM signal distortion only occurs in the yielding stage.

Inspired by the above researches and progress in the MMM method, we conducted research in order to better promote the application and development of MMM detection technology for steel bar stress detection in bridge engineering. On the basis of the SMFL signal variation law of bare steel bars that was studied before [41], in this paper, the specimens of steel bars covered by concrete (SBCC) with different concrete strengths and different steel bar diameters are used as test pieces. The variation law of SMFL on the surfaces of the SBCC specimens during the static loading process by the electronic universal testing machine (EUTM) is studied. According to the test results, an analysis and comparison with the rare steel bar are made.

\section{Materials and Methods}

The specimen studied in this paper is the SBCC specimen, as shown in Figure 2. The total length of the SBCC specimen is $500 \mathrm{~mm}$, which consists of an outer layer of concrete and the internal thread steel bar. The outer concrete section is located at the middle of the axial direction of the steel bar, of which the design length is $300 \mathrm{~mm}$. When making the specimen, a bare steel bar length of $100 \mathrm{~mm}$ is reserved at both ends of the specimen so that the EUTM can hold the force during the static loading process. The concrete section of the SBCC specimen has a square cross-section. The SBCC specimen cross-section is composed of two parts: One is the outer concrete layer of $35 \mathrm{~mm}$ thickness, and the other is the section of the steel bar cross-section with different diameters, as shown in Figure 2c. The steel bar wrapped by the outer concrete is an HRB400 threaded steel bar. The steel bar is located in the middle of the cross-section of the specimen, and its total length is $500 \mathrm{~mm}$. In the SBCC specimens of the test, five kinds of steel bar diameters were used, which were 12, 16, 18, 20, and $25 \mathrm{~mm}$. The outer concrete of the specimens contained three strengths, namely C30, C40, and C50. The chemical composition and mechanical properties of the steel bars are shown in Table 1, and the concrete mix ratio is shown in Table 2. Under the concrete of each strength, four SBCC specimens of the same diameter and size were fabricated, and the numbers in the group were 1\#, 2\#, 3\#, and 4\#. The SBCC specimen number naming rule is "diameter-concrete strength-in-group number". For example, the first specimen in the group, whose steel bar diameter is $25 \mathrm{~mm}$ and outer concrete strength is C30 strength, is numbered "Ф25-C30-1". There were 60 SBCC specimens in the test in total. As shown in Table 3, the SBCC 
specimens were recorded according to the differences of the internal steel bar diameters and the strengths of the concrete. They were divided into 15 groups, totaling 60 specimens, with each group containing four identical steel bars with the same diameter and the same-strength concrete. In the test, the 1\# SBCC specimen in each group was used to test the variation of the SMFL signal as the tensile force changed, and the tensile-displacement curve of the SBCC specimen during the loading process was also tested. The 2\# SBCC specimen was used to test the variation of the SMFL signal along the axial position of the specimen under different tensile forces. The $3 \#$ and $4 \#$ specimens were spare specimens designed for the 1\# and 2\# specimens, respectively. Since the specimen preparation cycle is long, there are two designed spare specimens.

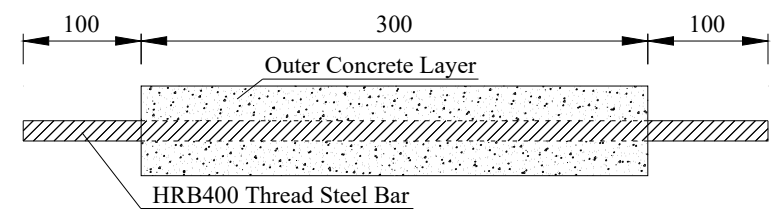

(a) The Elevation of the SBCC Specimen

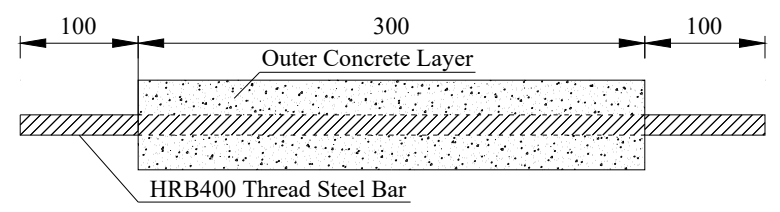

(b) The Floor Plan of the SBCC Specimen

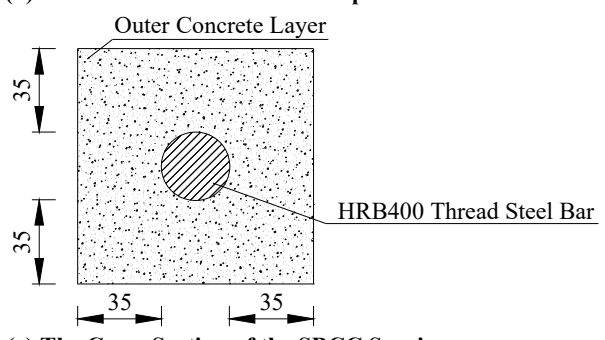

(c) The Cross Section of the SBCC Specimen

Figure 2. Steel bar covered by concrete (SBCC) specimen size design (unit: $\mathrm{mm}$ ). (a) The elevation of the SBCC specimen, (b) the floor plan of the SBCC specimen, and (c) the cross section of the SBCC specimen.

Table 1. Chemical composition and mechanical parameters of steel bars.

\begin{tabular}{ccccccc}
\hline \multirow{2}{*}{ Type of Steel } & \multicolumn{4}{c}{ Mass Fraction Chemical Composition (\%) } & \multirow{2}{*}{ Designed Tensile Strength (MPa) } \\
\cline { 2 - 5 } & $\mathbf{C}$ & $\mathbf{S i}$ & $\mathbf{M n}$ & $\mathbf{P}$ & $\mathbf{S}$ & \\
\hline HRB 400 & 0.2 & 0.4 & 1.3 & 0.03 & 0.02 & 360 \\
\hline
\end{tabular}

Table 2. SBCC specimens' concrete mix ratio.

\begin{tabular}{cccccccc}
\hline \multirow{2}{*}{ Concrete Mark } & \multicolumn{5}{c}{ Material Usage Per Cubic Concrete (kg) } & \multirow{2}{*}{ Volumetric Weight $\left(\mathbf{k N} / \mathbf{m}^{\mathbf{3}}\right)$} \\
\cline { 2 - 6 } & Water & Cement & Sand & Crushed Stone & Admixture & Fly Ash & \\
\hline C30 & 178 & 334 & 759 & 1092 & 5.3 & 41 & 24.09 \\
C40 & 170 & 391 & 718 & 1083 & 7.3 & 41 & 24.10 \\
C50 & 173 & 410 & 696 & 1083 & 8.2 & 50 & 24.20 \\
\hline
\end{tabular}

Table 3. SBCC specimens concrete mix ratio.

\begin{tabular}{ccccccc}
\hline \multirow{2}{*}{ Concrete Mark } & \multicolumn{5}{c}{ Diameter of Steel Bar } & \multirow{2}{*}{ Total } \\
\cline { 2 - 6 } & $\mathbf{1 2} \mathbf{~} \mathbf{m m}$ & $\mathbf{1 6} \mathbf{~} \mathbf{m}$ & $\mathbf{1 8} \mathbf{~} \mathbf{m}$ & $\mathbf{2 0} \mathbf{~} \mathbf{m}$ & $\mathbf{2 5} \mathbf{~} \mathbf{m}$ & \\
\hline C30 & 4 & 4 & 4 & 4 & 4 & 20 \\
C40 & 4 & 4 & 4 & 4 & 4 & 20 \\
C50 & 4 & 4 & 4 & 4 & 4 & 20 \\
Total & 12 & 12 & 12 & 12 & 12 & 60 \\
\hline
\end{tabular}


The instrument used for static loading in this paper is the EUTM (DNS300, Changchun Research Institute for Mechanical Science Co., Ltd., Changchun, China). The clamping diameter that can be matched by the EUTM clamps at both ends already includes the diameters of the steel bars in all SBCC specimens in this test (minimum $12 \mathrm{~mm}$, maximum $25 \mathrm{~mm}$ ). As shown in Figure 3, the SBCC specimen numbered $\Phi 12-C 30-2$ is clamped on the EUTM. The control and data acquisition of the EUTM are automatically set by a computer program, and can be loaded according to displacement and force. During the loading process, the EUTM can be kept under one displacement or one tension, and the time of holding and data acquisition frequency can be set artificially. The test in this paper is loaded by displacement, and the loading rate is $5 \mathrm{~mm} / \mathrm{min}$. The device for SMFL signal acquisition on the surfaces of SBCC specimens is the SMFL signal automatic acquisition device that was developed by us. As shown in Figure 4, the device is mainly composed of a self-assembled movable walking bracket, a magnetic sensor produced by Honeywell (Morris Plains, NJ, USA), and PC-side control software. The device can realize continuous automatic acquisition of SMFL signals at one point or one line segment in the space coordinate system.

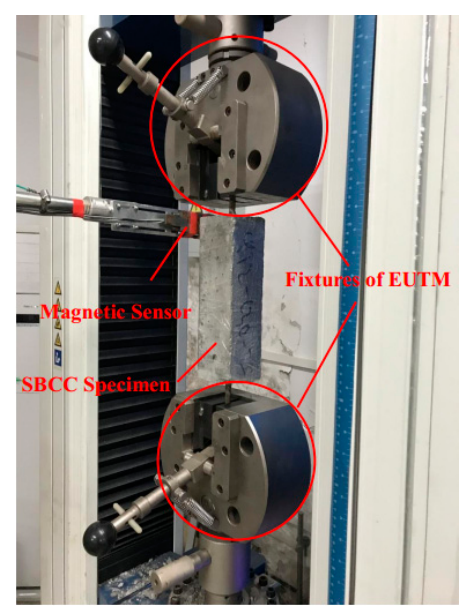

Figure 3. SBCC specimen and the electronic universal testing machine (EUTM) clamp picture.

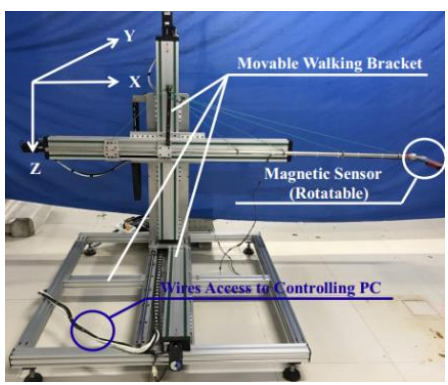

(a)

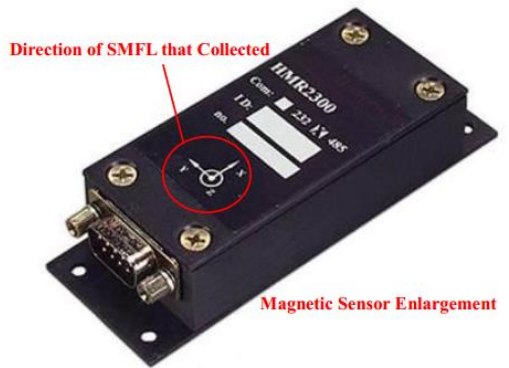

(b)

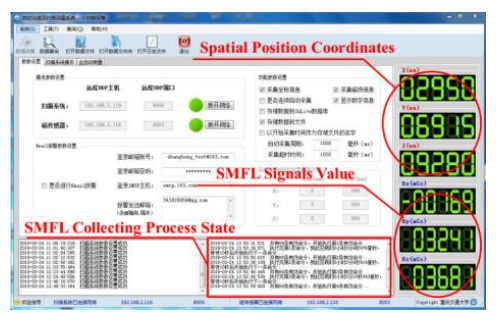

(c)

Figure 4. The self-magnetic flux leakage (SMFL) signal automatic acquisition device. (a) Overall equipment, (b) magnetic sensor enlargement, and (c) PC-side operating software interface. 
During the whole tensile loading process of the 1\# SBCC specimen in each specimen group, the magnetic sensor was placed at the midpoint $(1 \mathrm{~cm}$ above the surface) of the specimen along the length direction for measuring the variation of the SMFL signal with the tensile force as well as the tension-displacement curve of the specimen during the loading process. The EUTM starts to load from $0 \mathrm{kN}$ to the tensile force that breaks the internal steel bar of the SBCC specimen. The relevant test of each group's 1\# SBCC specimen is also referred to as the SMFL signal fixed-point monitoring test of the SBCC specimen [41]. According to the measured tension-displacement curves of the 1\# SBCC specimens in each group, one to five points are selected in each of the elastic stage, the yielding stage, the strengthening stage, and the necking stage. The selected points should be dispersed as evenly as possible in each loading stage, and the tensile forces corresponding to the points selected for the specimens with the same diameter of the steel bars should be as equal as possible. The loading displacements corresponding to these points are taken as the target loading displacements (TLDs) of the 2\# SBCC specimen in the same group. For example, one SBCC specimen selects P1, P2, P3, ..., P10 for a total of 10 TLD points in four stages, according to the tension-displacement curve. When the EUTM is loaded to the displacement corresponding to the P1 point, the current loading is maintained, keeping the displacement and the tension unchanged, and then the magnetic sensor of the SMFL signal automatic acquisition device scans the SMFL signal on the surface along the axial direction of the specimen. During the scanning acquisition process, the lift-off heights (LHs) of the magnetic sensor from the surface of the SBCC specimen are 1,3, and $5 \mathrm{~cm}$, respectively, as shown in Figure 5 . In order to avoid the influence of local magnetization caused by excessive clamping forces on the clamping parts at both ends of the SBCC specimen [42,43], the length of the magnetic sensor scanning acquisition path under each LH is $245 \mathrm{~mm}$ in the middle of the concrete section above the SBCC specimen. After the scanning and acquisition of the SMFL signals of all the paths corresponding to the loading displacement of the P1 point completing, the EUTM continues to load and cause tensile to the loading displacement corresponding to the P2 point, and the above scanning and collecting work is repeated until the scanning operation of the loading displacement corresponding to the P10 point ends. In this paper, the study of the variation of the SMFL signals on the surface of each group's 2\# specimen along the axial position of the specimen under different tensions is also referred to as the axial scanning test of the SBCC specimen [32,41]. In the test process, in order to prevent the outer concrete of the SBCC specimen getting splashed when it is broken, the specimen can be wrapped with a transparent tape. To avoid the formation of the hoop effect [44], the wrapping cannot be too tight.

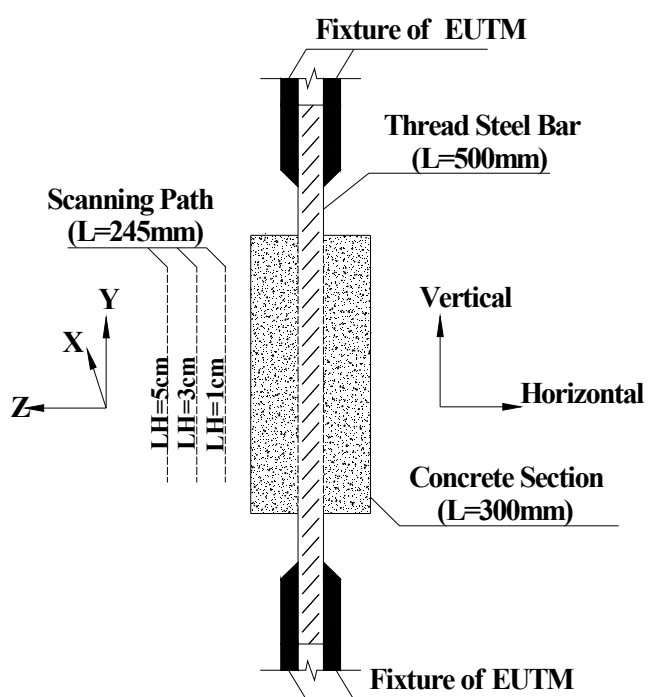

Figure 5. Schematic diagram of the scanning path of the magnetic sensor in the axial scanning test. 


\section{Results and Discussion}

\subsection{SMFL Signal Results of SBCC Specimens in the Fixed-Point Monitoring Test}

\subsubsection{The Variation Law of the Tangential Component (By Signal) of the SMFL Signal}

The top-half part of Figure 6 is the fixed-point monitoring test result of tangential components of the SMFL signals (By signals), and the bottom-half part is the tension-displacement curve of the Ф20-C40-1 SBCC specimen during the whole static loading process. Since the fixed-point monitoring test results and the tension-displacement curve performance are similar, only the test results of the Ф20-C40-1 SBCC specimen are shown here. On the tension-displacement curve, the SBCC specimen exhibits the same four stages of elastic, yielding, strengthening, and necking as the steel stress-strain curve. There are two differences on the shape between the tension-displacement curve of the SBCC specimen and the stress-strain curve of the steel. One is that in the stress-strain curve of the steel, the curve is smooth except for the yielding stage, and there is no local fluctuation. However, the curve has local fluctuations which are more frequent in the four stages of the SBCC specimen. Second, the stress-strain curve of the steel fluctuates horizontally with the increase of strain or loading displacement during the yielding stage, while the fluctuation of the SBCC specimen in the yielding stage is increasing fluctuation with the increase of the loading displacement. This phenomenon can be explained by the fact that the deformation of the steel bar is significantly increased while the deformation of the concrete is almost constant during the yielding stage, and the deformation difference between the two occurs. Due to the bonding property between the concrete and the steel bar, the required tensile force is greater than for the bare steel bar for deformation of the same size, as the deformation difference between the concrete and the steel bar needs to be overcome. On the tension-displacement curve, the local tension is frequently fluctuating, and the increased displacement can be explained by the fact that there is a dislocation between the steel bar and the concrete during the loading process.

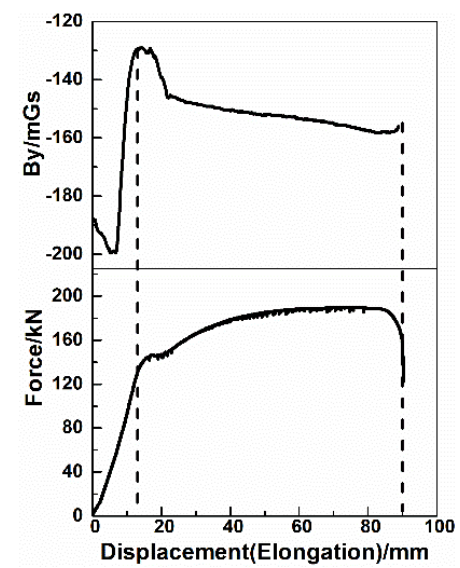

Figure 6. Variation of the By signals, and the tension-displacement curve during static loading of the Ф20-C40-1 specimen.

In order to simplify the calculation and to facilitate the quantification, the tensile force corresponding to the displacement at the midpoint of the yielding stage on the tension-displacement curve of the SBCC specimen is defined as the "yield tension" in this paper. As far as the By signal is concerned, there is a significant difference compared with the bare steel bar [41]. In the preliminary stage of loading, that is, when the EUTM starts loading, the value of the By signal becomes smaller and smaller. The diameters of the steel bars of the specimens are different, and the decreases in magnitude of the By signals are different, of which the range is $10-80 \mathrm{mGs}$. When the By signal is first reduced to a certain extent, a minimum extreme point appears on the By signal curve, and then the By signal increases until the "platform stage" appears on the curve of the By signal, with a maximum 
extreme point. From the minimum extreme point to the maximum extreme point, the increment in the magnitude of the By signal is in the range of 30-120 mGs. After entering the "platform stage", the variation of the By signal is small, which is $10-30 \mathrm{mGs}$. At the moment when the steel bar of the specimen is broken, there is no sudden change in the value of the By signal. At the initial loading stage of the bare steel bar, there is no phenomenon that causes the By signal to become smaller. On the curve of the By signal of the SBCC specimen, before entering the "platform stage", the maximum extreme point will still appear on the curve. The ratio of the tension corresponding to the maximum extreme point to the yield tension is $89.21 \%$ for the $12 \mathrm{~mm}$ diameter steel bar SBCC specimen, $89.26 \%$ for the $18 \mathrm{~mm}$ diameter steel bar SBCC specimen, and 89.65\% for the $20 \mathrm{~mm}$ diameter steel bar SBCC specimen. The ratio increases with the increase of the diameter of the steel bar of the SBCC specimen under the same concrete strength, and the ratio of the tension corresponding to the maximum extreme point to the yield tension is close to but less than $90 \%$ overall. The ratio of the tension corresponding to the maximum extreme point to the yield tension of the SBCC specimen is closer to the yielding stage compared to the ratio of the rare steel bar, which is $65 \%$. This difference can be explained by the fact that the outer concrete of the SBCC specimen shrinks during the pouring and curing period, and the deformation is inconsistent with the deformation of the inner steel bars, resulting in shrinkage stress, which shows tensile force to the concrete and pressure on the steel bar. The SMFL signal of the steel bar is a kind of weak magnetic signal, which is quite sensitive to the change of stress. After the concrete curing is completed, the surface of the steel bar has formed a kind of "pre-pressure". The direction of the SMFL signal caused by this "pre-pressure" is opposite to the tensile force. Therefore, at the initial stage of loading, the magnetic fields on the surface of the specimen cancel each other and the By signal decreases first and then increases. The maximum extreme point on the fixed-point monitoring test curve of the SBCC specimen is closer to the yield tension than that of the bare steel bar, which can be considered as being due to the "pre-pressure" generated by the outer concrete needing to be offset, and so the curve translates right.

\subsubsection{The Variation Law of the First Derivative of the By Signal}

The top-half part of Figure 7 is the first derivative curve of the By signal of the SBCC specimen, and the bottom-half part is still the tension-displacement curve of the SBCC specimen during the whole static loading process. Since the test results show similarity, only the test results of the specimen numbered $\Phi 20-C 40-1$ are shown here. From the top-half part of Figure 7, that is, the first derivative curve of the By signal, it can be seen that in the entire loading process of the SBCC specimen, one significant peak still occurs during the elastic stage but no peaks occur during the yielding stage. On the first derivative curve of the By signal, the portion at which the peak is removed exhibits a sawtooth fluctuation of a value of around 0 . Except for the value of one individual point fluctuation which is slightly larger (not exceeding 10), the remaining fluctuations are within five. At the moment when the steel bar of the SBCC specimen breaks, the first derivative curve of the By signal has a slight increase, of which the value is about five. The ratio of the tensile force corresponding to the peak point on the first derivative curve of the By signal to the yield tension that defined in this paper is $73.86 \%$ for the SBCC specimen with the diameter of $12 \mathrm{~mm}, 61.81 \%$ for the SPCC specimen with the diameter of $18 \mathrm{~mm}$, and $53.56 \%$ for the SPCC specimen with the diameter of $20 \mathrm{~mm}$, which denotes that under the same strength of the outer concrete, the ratio decreases as the diameter of the steel bar in the SBCC specimen increases. For the first derivative curve variation of the By signal of the SBCC specimen with the displacement loading process, compared with the bare steel bar [41], there is no obvious peak in the yielding stage. On the part except for the peak point of the first derivative curve of the By signal, the similarities between the two types of specimens are that the values are close to zero and fluctuate slightly around 0 , and the difference is that the curves of these parts for bare steel specimens are smooth and have no frequent fluctuations, while the curves of these parts for the SBCC specimen show a small amplitude of sawtooth fluctuations. The reason for the above difference on the first derivative curve of the By signal of the SBCC specimen can be explained by the fact that the dislocation between the steel 
bar and the concrete during the stretching process causes fluctuation of the tensile force in the steel bar, and the SMFL signal is sensitive to the change of the tensile force, thus also generating fluctuations.

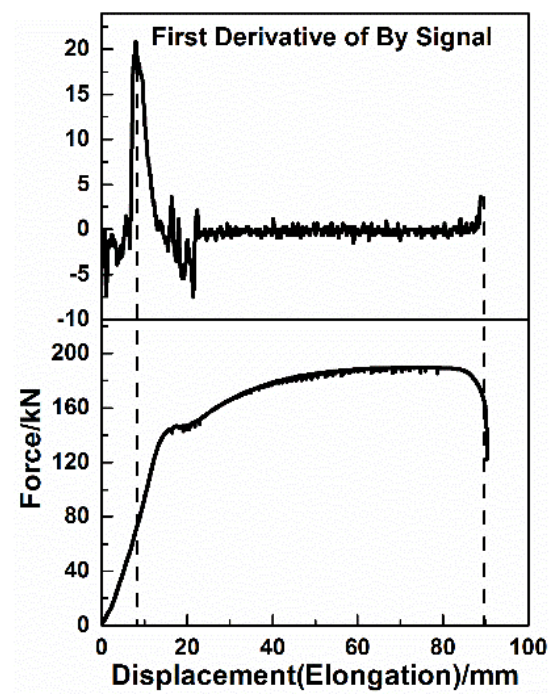

Figure 7. Variation of first derivative of the By signal, and tension-displacement curve of the $\Phi 20-C 40-1$ specimen during the loading process.

\subsection{The Results of the By Signal in the Axial Scanning Test of SBCC Specimens}

In the axial scanning test of SBCC specimens, the magnetic sensors were scanned and recorded along the axial position of the specimens at different LHs, as shown in Figure 8. In Figure 8, the horizontal axis of the coordinate is the positional coordinate of the SBCC specimen along the axial direction, and the ordinate is the size of the By signal value collected in this position. Since the results of the axial scanning test of the 2\# specimens are similar, and the TLDs of the EUTM are numerous, in order to more clearly show the trend of the curve of the axial scanning test results and the variation of the By signal along the axial position, only the SBCC specimen numbered $\Phi 25-C 30-2$ is shown here, of which the LH is $1 \mathrm{~cm}$ and the TLDs are 20,30 , and $50 \mathrm{~mm}$.

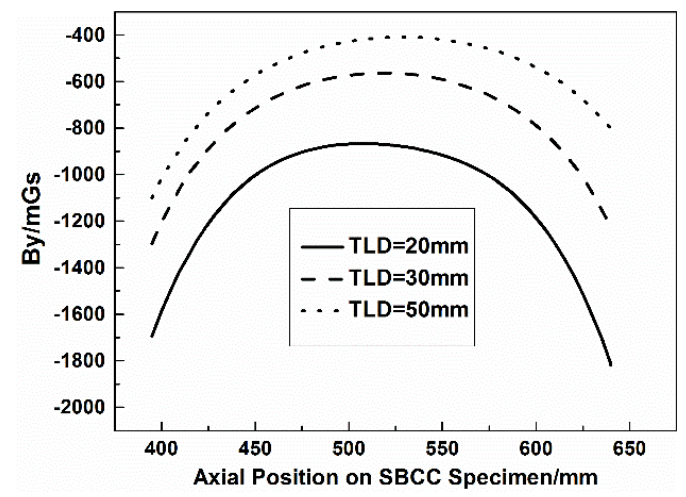

Figure 8. The axial scanning test results of SBCC specimen at lift-off heights $(\mathrm{LH})=1 \mathrm{~cm}$.

It can be seen from Figure 8 that the axial scanning test results of the SBCC specimen are similar to those of the bare steel bar, that is, the distribution of the By signal curve of the SBCC specimen in the scanning section shows that the values at both ends are small while the intermediate values are large. The minimum value of the whole scanning result curve also appears at one end of the two ends, and the maximum value appears in the middle, but not necessarily at the absolute midpoint of the curve. The values at the two ends of the curve are not necessarily the same. The value of the By signal is continuously and evenly transitioned from the two ends to the middle. There is no obvious 
jump phenomenon in the numerical value. The whole scan result curve is very smooth and there is no fluctuation in the local values. The By signals at both ends of the curve are always smaller than the value at the middle of the curve, which can be explained by the end effect of the specimen.

\subsubsection{Verification of the Parameter $A_{\mathrm{T}}$}

For the SMFL signal of a bare steel bar, we proposed the "force-induced magnetic fluctuation parameter" $A_{\mathrm{T}}$ in reference [41], which is calculated as follows:

$$
A_{\mathrm{T}}=\ln \left(\frac{\mathrm{By}_{\max }-\mathrm{By}_{\min }}{b_{\mathrm{y}}}\right)
$$

where $B y_{\max }$ is the largest value on the axial scanning test curve of $B y, B y_{\min }$ is the minimum value of By on the curve, and $b_{\mathrm{y}}$ is the unit vector of the SMFL signal of the bare steel bar along the $y$-axis direction, of which the size is $1 \mathrm{mGs}$. The larger the value of $A_{\mathrm{T}}$, the greater the fluctuation of the By signal along the axial direction. According to the same method, the $A_{\mathrm{T}}$ values of the SBCC specimens were calculated by the same method, and the $A_{\mathrm{T}}-T$ diagram was made. The results show that there is also a linear relationship between $A_{\mathrm{T}}$ and $T$ in the linear elastic stage of the $A_{\mathrm{T}}-T$ diagram of the SBCC specimen. The 2\# SBCC specimens of each group are summarized in the linear portion of the $A_{\mathrm{T}}-T$ diagram under different LHs, as shown in Figure 9.
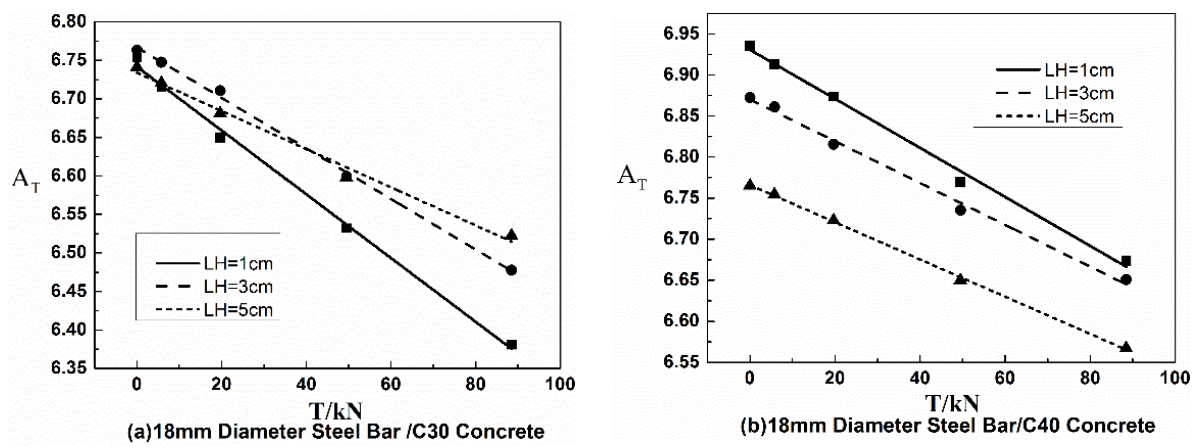

Figure 9. Linear portions of $A_{\mathrm{T}}-T$ diagrams with different strengths of outer concrete and different steel bar diameter specimens, at different LHs.

Figure 9 is a summary view of the linear portion of the $A_{\mathrm{T}}-T$ diagram of the $18 \mathrm{~mm}$-diameter steel bar specimens under the concrete strengths of C30 and C40, of which the LHs are 1, 3, and $5 \mathrm{~cm}$, respectively. Since the test results are similar, only the linear portion of the $A_{\mathrm{T}}-T$ diagram of the $18 \mathrm{~mm}$-diameter steel bar specimen under the strengths of the above-mentioned outer concrete are listed here. It can be seen from Figure 9 that the linear portion of the $A_{\mathrm{T}}-T$ diagram of the SBCC specimen monotonously decreases as the internal tensile force $T$ of the specimen increases, and the linear relationship between the two is significant. For the SBCC specimens with the same concrete strength and the same steel bar diameter, the slope of the linear segment of the $A_{\mathrm{T}}-T$ diagram increases numerically as the LH of the magnetic sensor increases in the axial scanning test. This indicates that in the linear elastic stage of the SBCC specimen, the tensile force can still be calculated using the method of the $A_{\mathrm{T}}-T$ diagram of the bare steel bar as proposed in Reference [41].

\subsubsection{Strengthening Stage Tensile Force Calculation and Area Ratio Deviation Parameter $S_{\mathrm{D}}$}

From the SBCC specimen axial scanning test curve, the $A_{\mathrm{T}}-T$ diagram method can only be applied to calculate the tensile force of the specimen in the linear elastic stage, and it is not applicable to the tensile force calculation for other stress stages. By observing the trend of the axial scanning test curve along the axial position of the specimen, as shown in Figure 8, the values of the two ends are small while the intermediate values are large, and the numerical values at both ends are not equal. In theory, 
the steel bar can be regarded as a magnet with a weak external magnetic field after being magnetized by the tensile force and the geomagnetic field. Therefore, the curve of the axial scanning test should be a symmetrical curve, with the values of the two ends on the curve being equal in magnitude, and the maximum value appearing in the absolute middle part on the curve.

As shown in Figure 10, the curve $\widehat{\mathrm{P} 1 \mathrm{P} 2 \mathrm{P} 3}$ is an actual axial scanning test curve, where P1 is the left end point, P2 is the point where the maximum value of the By signal appears on the curve, and P3 is the right end point of the curve. The right-angled triangle P1P2O1 with the area S1 can be made with the line segment P1P2 as the hypotenuse, and the $S 2$ right-angled triangle P2P3O2 can be made with the P2P3 as the hypotenuse. In theory, S1 and S2 should be equal, and in the SBCC specimen axial scanning test, when TLD is equal to 0 , both $S 1$ and $S 2$ are very close in size. In the strengthening stage, the difference between $S 1$ and $S 2$ becomes larger. Based on this, this paper proposes an "area ratio deviation parameter" $S_{\mathrm{D}}$ which can reflect the asymmetry of the axial scanning test curve during loading. The specific calculation method is as follows:

$$
S_{D}=1-\frac{\min \{S 1, S 2\}}{\max \{S 1, S 2\}}
$$

where $S 1$ represents the area of a right-angled triangle with the hypotenuse connecting the left end point P1 of the curve to the point P2 of the maximum value, and S2 represents the area of a right-angled triangle with the hypotenuse connecting the right end point P3 of the curve to the point P2 of the maximum value. In the theoretical case, $S_{D}=0$. In the case of TLD $=0 \mathrm{~mm}$ or a quite small value, $S_{\mathrm{D}}$ is very close to 0 . The larger the TLD, especially in the later period of loading process, the farther $S_{\mathrm{D}}$ is from 0 . By calculating the $S_{\mathrm{D}}$ of each group of specimens under different TLDs, taking the internal tensile force of the SBCC specimen as the abscissa, and then calculating $S_{\mathrm{D}}$ as the ordinate, the $S_{\mathrm{D}^{-}}-T$ diagrams of the axial scanning test curves for different SBCC specimens can be made, as shown in Figure 11.

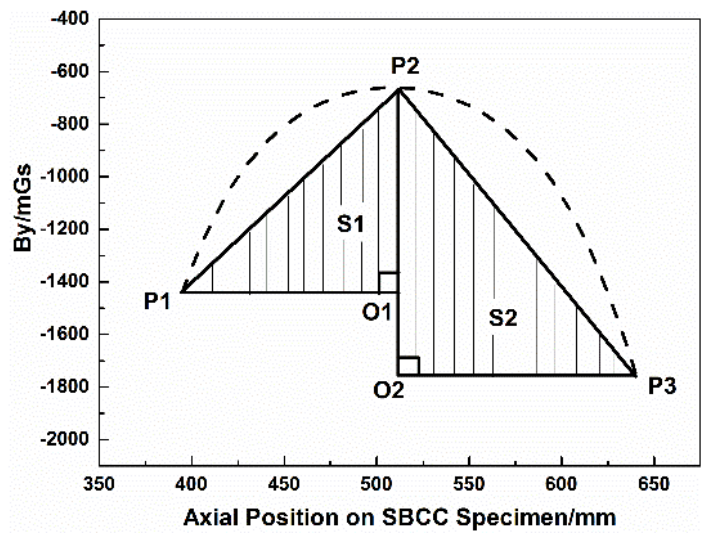

Figure 10. Schematic diagram of the values of the area ratio deviation parameter $S_{D}$.

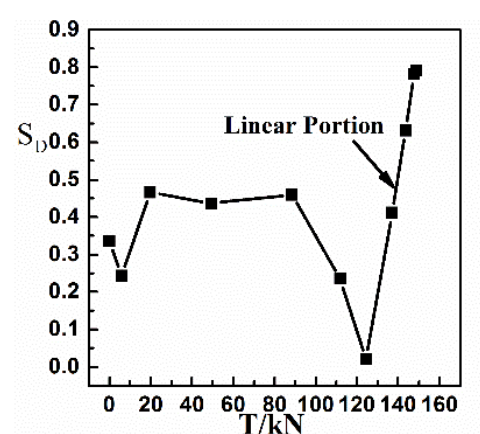

(a) $18 \mathrm{~mm}$ Steel Bar/ C30 Concrete

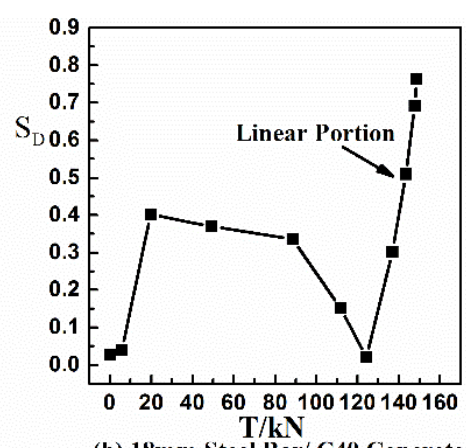

(b) $18 \mathrm{~mm}$ Steel Bar/ C40 Concret

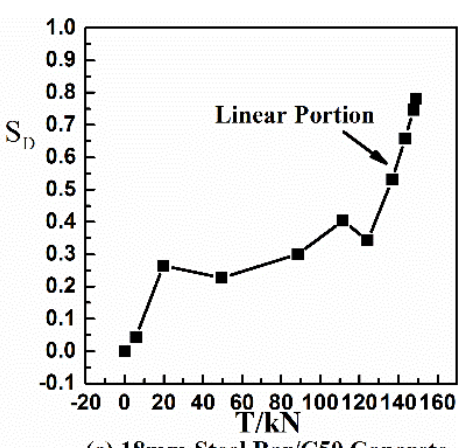

(c) $18 \mathrm{~mm}$ Stecl Bar/C50 Concrete

Figure 11. $S_{\mathrm{D}}-T$ diagrams of the axial scanning test curves for the SBCC specimens. 
Figure 11 shows the $S_{D^{-}}-T$ diagrams of the axial scanning test curves for the SBCC specimens numbered $\Phi 18-C 30-2, \Phi 18-C 40-2$, and $\Phi 18-C 50-2$. Since all SBCC specimens exhibit similar patterns in $S_{\mathrm{D}}-T$ diagrams, only the $S_{\mathrm{D}}-T$ diagrams of the above SBCC specimens at $\mathrm{LH}=1 \mathrm{~cm}$ are shown here. As shown in the figure, at the initial stage of loading, the $S_{\mathrm{D}}$ values of specimens increase with the increase of $T$, and this increase is large but no more than 0.5 , except for some individual specimens which have decreases less than 0.15 . When loading enters the yielding stage, the change of $S_{\mathrm{D}}$ with $T$ is relatively random, with some decreases with the increase of $T$ and some increases with the increase of $T$, and some specimens having decreases first and then increases with the increase of $T$, but the overall change does not exceed 0.2. After entering the strengthening stage, $S_{\mathrm{D}}$ monotonously increases with the increase of $T$, and the growth range is between 0.5 and 0.8 . The $S_{\mathrm{D}}$ values of the axial scanning test curves of all SBCC specimens do not exceed 0.85. In the strengthening stage, there is a significant linear relationship between $S_{\mathrm{D}}$ and $T$. As shown in the linear portion of Figure 11, since the selected TLD points in the linear portion of the figure already covers the entire strengthening stage, the linear relationship between $S_{\mathrm{D}}$ and $T$ can be used to calculate the internal tensile force of the SBCC specimen in the strengthening stage. The calculation method is as follows:

$$
\begin{gathered}
S_{\mathrm{D}}=K \cdot T+C \\
1-\frac{\min \{S 1, S 2\}}{\max \{S 1, S 2\}}=K \cdot T+C \\
1-\frac{\min \{S 1, S 2\}}{\max \{S 1, S 2\}}-C=K \cdot T \\
T=\frac{1-C-\frac{\min \{S 1, S 2\}}{\max \{S 1, S 2\}}}{K}
\end{gathered}
$$

where $K$ and $C$ are the slope and the longitudinal intercept of the linear portion in $S_{D^{-}}-T$ diagram of the specimens in strengthening stage, respectively, and $S 1$ and $S 2$ are the squares of the two right triangles obtained by the method from Figure 10 from the axial scanning test curve, respectively. According to the same method, the values of the axial scanning test curves of the different groups of the SBCC specimens are calculated, and the $S_{\mathrm{D}}-T$ diagram can be made. The specimens of the same outer concrete strength and the same steel bar diameter group are summarized in the linear portions of the $S_{\mathrm{D}}-\mathrm{T}$ diagrams at different LHs, as shown in Figure 12. Figure 12 is a summary view of the linear portions of the $S_{\mathrm{D}}-T$ diagrams of the 16,18, and $20 \mathrm{~mm}$-diameter steel bar specimens at LHs of 1,3 , and $5 \mathrm{~cm}$ when the outer concrete strengths are C30, C40, and C50, respectively. Since the $S_{\mathrm{D}}-T$ diagrams of the By signals of the SBCC specimens are similar in the linear portions during the strengthening stage, only the SBCC specimens of the above-mentioned steel bar diameters and the concrete of the above-mentioned strengths are listed here $(18 \mathrm{~mm}$-diameter steel bar specimens are shown with outer concrete strengths of C30, C40, and C50, and the remaining two diameters of steel bar diameter are only listed here with an outer concrete strength of C40). It can be seen from the figure that when the loading tensile force is in the strengthening stage of the specimen, the $S_{\mathrm{D}}$ value calculated with different TLDs increases monotonically with the increase of the internal tensile force $T$, and $S_{\mathrm{D}}$ shows a very significant linear relationship with $T$. The growth of the parameter $S_{\mathrm{D}}$ in the strengthening stage is relatively large, ranging from 0.5 to 0.8 . When the strength of the outer concrete is the same, the slope of the linear portion in the $S_{D^{-}} T$ diagram decreases as the diameter of the steel bar inside the SBCC specimens increases. When the steel bar diameters of the SBCC specimens are the same, the slope of the linear portion in the $S_{\mathrm{D}}-T$ diagram decreases as the strength of the outer concrete of the specimen increases. In the axial scanning test process of the same specimen, the slope of the linear portion in the $S_{D^{-T}}-T$ diagram decreases as the magnetic sensor scanning path LH increases. 

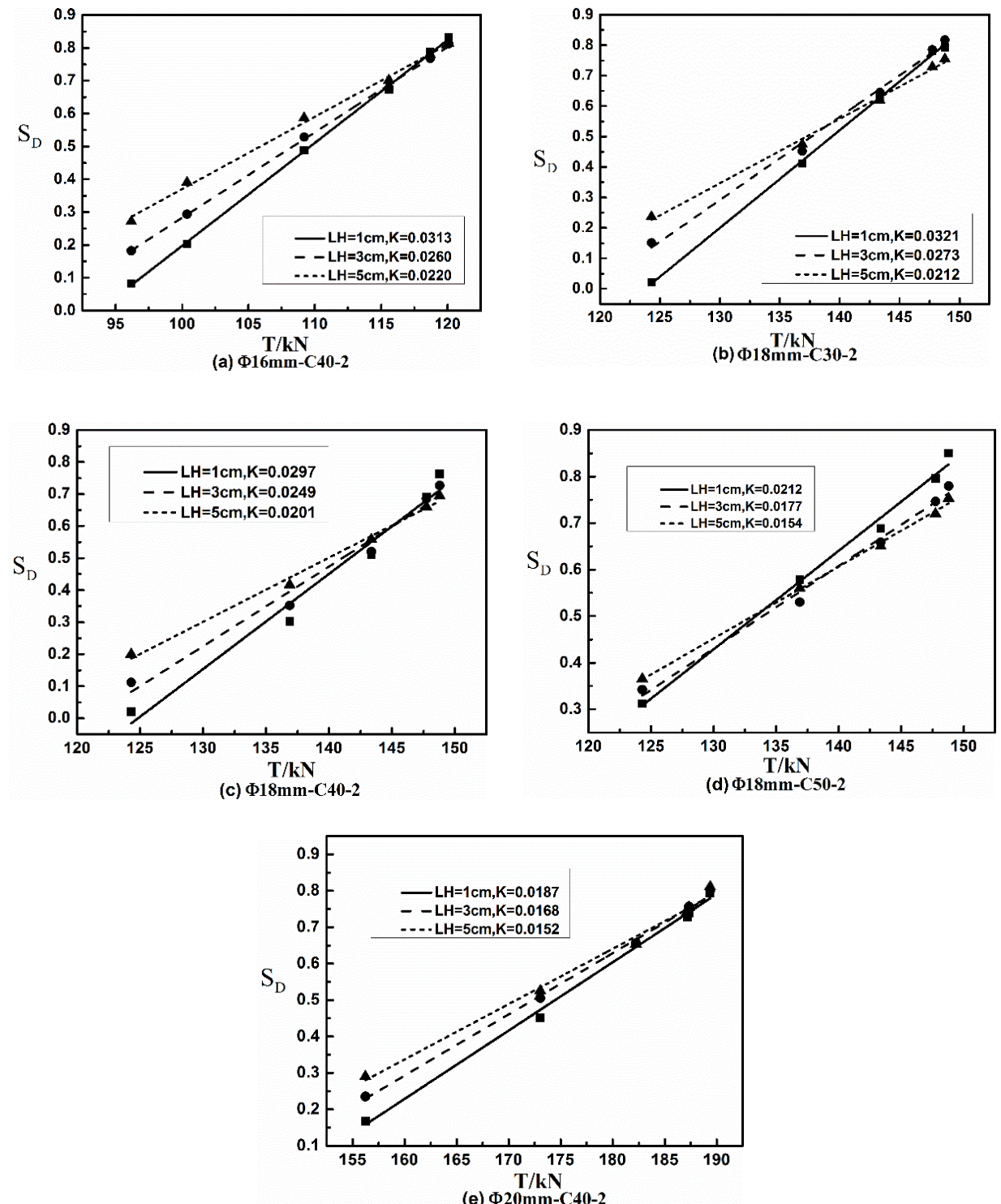

Figure 12. The linear portions of $S_{\mathrm{D}}-T$ diagrams of different strength of outer concrete and different steel bar diameter specimens, at different LHs. (a) $\Phi 16-C 40-2$, (b) $\Phi 18-C 30-2$, (c) $\Phi 18-C 40-2$, (d) $\Phi 18-C 50-2$, (e) $\Phi 20-C 40-2$.

\subsection{Influence of Outer Concrete Strength and Steel Bar Diameter of SBCC Specimens on Test Results}

After a total of 60 SBCC specimens that divided into 15 groups were investigated by the SMFL signal fixed-point monitoring test and the axial scanning test, it could be found that under different diameters of steel bars and different strengths of outer concrete conditions, the test results were not the same. In order to study the influences of different steel bar diameters and different outer concrete strengths on the test results, these two influencing factors were proposed separately, and the law was studied by controlling the variables. Since the test results showed similar laws, when studying the influence of the steel bar diameter on the test results, only the test results of the specimens with the outer concrete strength of $\mathrm{C} 40$ are listed here. When studying the influence of the strength of the outer concrete on the test results, only the test results for the SBCC specimens with a steel bar diameter of 18 $\mathrm{mm}$ are listed.

Figure 13a is a graph showing the variation of the tensile force corresponding to the maximum extreme point of the By signal before the yielding stage of the SBCC specimens in the fixed-point monitoring test. It can be seen from the figure that the tensile force corresponding to the maximum 
extreme point on the By signal curve is $47.3 \mathrm{kN}$ when the steel bar diameter of the specimen is $12 \mathrm{~mm}$, $91.2 \mathrm{kN}$ when the diameter is $16 \mathrm{~mm}, 100.9 \mathrm{kN}$ when the diameter is $18 \mathrm{~mm}, 130.6 \mathrm{kN}$ when the diameter is $20 \mathrm{~mm}$, and $193.8 \mathrm{kN}$ when the diameter is $25 \mathrm{~mm}$. That is, before the specimen enters the yielding stage, the magnitude of the tensile force corresponding to the maximum extreme point increases as the diameter of the steel bar of the SBCC specimen increases. Calculate the ratio of this tensile force corresponding to the yield tension defined in this paper, as shown in Figure 13b, which was $89.21 \%$ for the specimen with a steel bar diameter of $12 \mathrm{~mm}, 89.25 \%$ for the $16 \mathrm{~mm}$ specimen, $89.26 \%$ for the $18 \mathrm{~mm}$ specimen, $89.65 \%$ for the $20 \mathrm{~mm}$ specimen, and $89.82 \%$ for the $25 \mathrm{~mm}$ specimen. This ratio increases as the diameter of the steel bar inside the SBCC specimen increases, while it decreases as the diameter of the steel bar increases for the rare steel bar specimens [41].

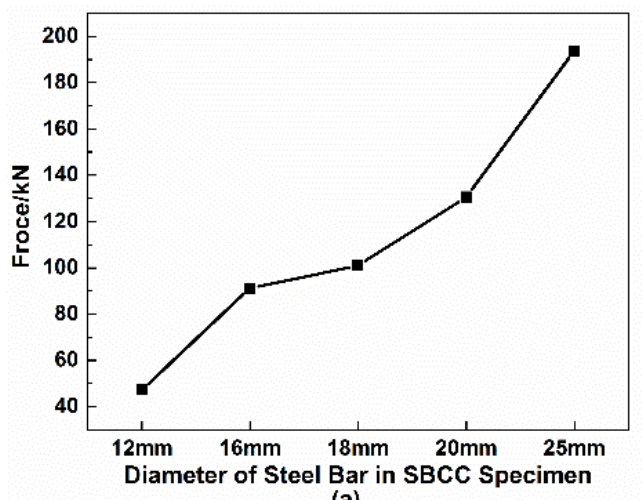

(a)

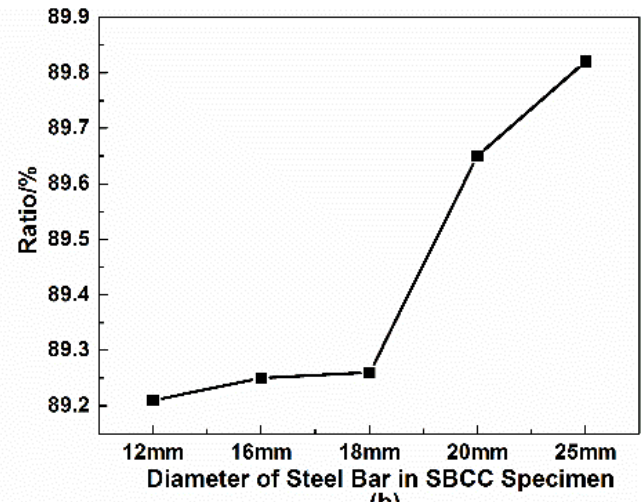

(b)

Figure 13. The tensile force corresponding to the maximum extreme point on the By curve in the fixed-point monitoring test, with different steel bar diameters. (a) Tensile force corresponding to the maximum extreme point, and (b) ratio of the tensile force at the maximum extreme point to the yielding tension.

As shown in Figure 14, the ratio of the tensile force corresponding to the peak value on the first derivative curve of the By signal to the yield tension in the fixed-point monitoring test is $74.6 \%$ for the $12 \mathrm{~mm}$-diameter steel bar specimen, $66.3 \%$ for the $16 \mathrm{~mm}$-diameter specimen, $61.8 \%$ for the $18 \mathrm{~mm}$-diameter specimen, $50.8 \%$ for the $20 \mathrm{~mm}$-diameter specimen, and $48.2 \%$ for the $25 \mathrm{~mm}$-diameter specimen. The ratio decreases with the increase of the steel bar diameter of the SBCC specimen.

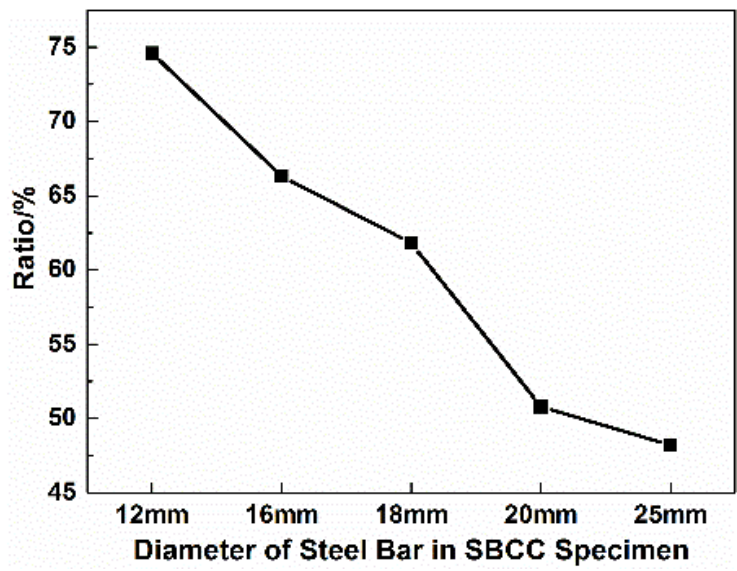

Figure 14. The ratio of the tensile force corresponding to the peak value on the first derivative curve of the By signal to the yield tension, with SBCC specimens of different steel bar diameters during the elastic stage in the fixed-point monitoring test. 
As shown in Table 4, it can be known that when the strength of the outer concrete of the SBCC specimen is $C 40$, the slope of the linear portion in the $S_{D^{-}}-T$ diagram decreases with the increase of the diameter of the steel bar in the specimen. When the diameter of the steel bar in the SBCC specimens is $18 \mathrm{~mm}$, the slope of the linear portion in the $S_{D^{-}}-T$ diagram of the SBCC specimen shows a decreasing trend as the strength of the outer concrete increases.

Table 4. Linear section slopes of the $S_{\mathrm{D}}-T$ diagrams of SBCC specimens with different strengths of outer concrete and different-diameter steel bars.

\begin{tabular}{|c|c|c|c|c|c|}
\hline \multirow{2}{*}{ LH } & \multicolumn{5}{|c|}{ Diameter of Steel Bar/The Outer Concrete Mark } \\
\hline & $16 \mathrm{~mm} / \mathrm{C} 40$ & $18 \mathrm{~mm} / \mathrm{C} 30$ & $18 \mathrm{~mm} / \mathrm{C} 40$ & $18 \mathrm{~mm} / \mathrm{C} 50$ & $20 \mathrm{~mm} / \mathrm{C} 40$ \\
\hline $1 \mathrm{~cm}$ & 0.0313 & 0.0321 & 0.0297 & 0.0212 & 0.0187 \\
\hline $3 \mathrm{~cm}$ & 0.0260 & 0.0273 & 0.0249 & 0.0177 & 0.0168 \\
\hline $5 \mathrm{~cm}$ & 0.0220 & 0.0212 & 0.0201 & 0.0154 & 0.0152 \\
\hline
\end{tabular}

\section{Conclusions}

In order to study the stress detection by the MMM method in more depth, this paper studied the SBCC specimens based on the study of bare steel bars. Research on the variation law of the SMFL signal with the tensile force of the specimen, and the relationship between the SMFL signal and the axial position along the specimen under different tensile forces was carried out, and the following conclusions can be drawn:

(1) In the fixed-point monitoring test of the SBCC specimen, the tangential component (By signal) of the SMFL signal on the surface of the specimen first decreases and then increases during the loading process. When it increases to a certain size, it will enter the "platform stage". The By signal curve still has a maximum extreme point before entering the "platform stage", and the ratio of the tensile force corresponding to the maximum extreme point to the yield tension is close to but less than $90 \%$. The first derivative curve of the By signal shows a distinct peak during the elastic stage of the specimen, and no significant peaks appear during the yielding stage.

(2) In the axial scanning test of the SBCC specimen, the By signal is distributed along the axial position of the specimen to a curve with small values at both ends and a large value in the intermediate part, of which the local region is smooth without fluctuations or numerical jumps. In order to calculate the internal tensile force of the specimen effectively and accurately, this paper proposes the "area ratio deviation parameter" $S_{\mathrm{D}}$. This parameter shows a significant linear relationship with the internal tensile force $T$ of the specimen during the strengthening stage of the SBCC specimen, which can provide a new method for the tensile force calculation of the steel bar in the strengthening stage.

(3) Regarding the influences of the steel bar diameter of the SBCC specimen and the strength of the outer concrete on the test results, before the specimen enters the yielding stage, the corresponding tensile force of the maximum extreme point increases with the increase of the diameter of the steel bar. The ratio of the tensile force to the yielding tension increases as the diameter of the steel bar in the SBCC specimen increases. The ratio of the tensile force corresponding to the peak value on the first derivative curve of the By signal to the yield tension decreases with the increase of the steel bar diameter of the SBCC specimen. The slope of the linear portion in the $S_{D^{-}} T$ diagram decreases with the increase of the diameter of the steel bar in the specimen, and it shows a decreasing trend as the strength of the outer concrete increases.

(4) Based on the theory of metal magnetic memory, the experimental research object in this paper is closer to the actual project, and a new internal force calculation method has been proposed. The method is simple in principle, easy in operation, and rapid in detection. It can supplement the existing steel bar stress detection methods, and has prospective research value. 
Author Contributions: Funding acquisition, C.P., J.Z., and R.Z.; investigation, C.P. and R.Z.; methodology, C.P. J.Z., and R.Z.; project administration, J.Z. and R.Z.; validation, C.P., Q.Z., Z.C., and Y.Z.; writing-original draft, C.P.; writing-review \& editing, J.Z. and R.Z.

Funding: This research was funded by the National Key Research and Development Program of China (2016YFC0802202), the National Science Fund for Distinguished Young Scholars (51425801), the National Natural Science Foundation of China (51808082), the Science and Technology Research Project of Chongqing Science \& Technology Bureau of China (cstc2017rgzn-zdyfX0018, cstc2018jscx-mszdX0084), the Open Fund of Engineering Research Center of Bridge Structure and Material in the Mountainous Area of Ministry of Education of China in Chongqing Jiaotong University (QLGCZX-JJ2016-7), and the Chongqing Jiaotong University Graduate Education Innovation Fund (2018S0123).

Conflicts of Interest: The authors declare no conflict of interest.

\section{References}

1. Gardner, D.; Lark, R.; Jefferson, T.; Davies, R. A survey on problems encountered in current concrete construction and the potential benefits of self-healing cementitious materials. Case Stud. Constr. Mater. 2018, 8, 238-247. [CrossRef]

2. Hobbs, D.W. Concrete deterioration: Causes, diagnosis, and minimising risk. Int. Mater. Rev. 2001, 46, 117-144. [CrossRef]

3. Zhou, L. A historical review and the state of art of the durability of bridge structures. Bridge Constr. 2000, $4,58-61$.

4. He, S.H.; Zhao, X.M.; Ma, J.; Zhao, Y.; Song, H.S.; Song, H.X.; Cheng, L.; Yuan, Z.Y.; Huang, F.W.; Zhang, J.; et al. Review of highway bridge inspection and condition assessment. China J. Highway Transp. 2017, 30, 63-80.

5. Xu, C.; Song, W.; Pan, Q.; Li, H.; Liu, S. Nondestructive testing residual stress using ultrasonic critical refracted longitudinal wave. Physics Procedia 2015, 70, 594-598. [CrossRef]

6. Rossini, N.S.; Dassisti, M.; Benyounis, K.Y.; Olabi, A.G. Methods of measuring residual stresses in components. Mater. Des. 2012, 35, 572-588. [CrossRef]

7. Liu, L.Y.; Deng, S.W.; Yang, S.X. Nondestructive testing technique for residual stress by using eddy current thermography and its application. Nondestr. Test. 2018, 40, 5-9.

8. Pan, Q.; Shao, C.; Xiao, D.; Pan, R.; Liu, X.; Song, W. Robotic ultrasonic measurement of residual stress in complex curved surface components. Appl. Bionics Biomech. 2019, 1, 2797896. [CrossRef]

9. Zheng, Z.; Lei, Y.; Xue, X. Numerical simulation of monitoring corrosion in reinforced concrete based on ultrasonic guided waves. Sci. World J. 2014, 1, 752494. [CrossRef]

10. Habibalahi, A.; Habibalahi, M.; Samadian, K. Pulsed eddy current applied to measure residual stress in welding. J. Test. Eval. 2018, 46, 2623-2629. [CrossRef]

11. Jomdecha, C.; Cai, W.; Xie, S.; Chen, Z.; Li, P. A numerical study on eddy current signal characteristics of imitative stress corrosion cracks. Int. J. Appl. Electromagnet Mech. 2017, 55, 257-269. [CrossRef]

12. Fu, X.S.; Niu, Z.Q.; Deng, Y.; Zhang, J.; Liu, C.; Chen, G.; Li, Z.; Zhou, W. Accuracy of X-ray diffraction measurement of residual stresses in shot peened titanium alloy samples. Surf. Coat. Technol. 2019, 361, 364-370. [CrossRef]

13. Turan, M.E.; Aydin, F.; Sun, Y.; Cetin, M. Residual stress measurement by strain gauge and X-ray diffraction method in different shaped rails. Eng. Fail. Anal. 2019, 96, 525-529. [CrossRef]

14. Xing, H.Y.; Xu, M.Q.; Wang, R.; Zhou, J.Z. MMM fatigue damage evaluation and life prediction modeling. Key Eng. Mater. 2006, 324, 619-622. [CrossRef]

15. Lu, Y.; Li, J.; Ye, L.; Wang, D. Guided waves for damage detection in rebar-reinforced concrete beams. Constr. Build. Mater. 2013, 47, 370-378. [CrossRef]

16. Karayannis, C.G.; Voutetaki, M.E.; Chalioris, C.E.; Providakis, C.P.; Angeli, G.M. Detection of flexural damage stages for RC beams using piezoelectric sensors (PZT). Smart Struct. Syst. 2015, 15, 997-1018. [CrossRef]

17. Karayannis, C.G.; Chalioris, C.E.; Angeli, G.M.; Papadopoulos, N.A.; Favvata, M.J.; Providakis, C.P. Experimental damage evaluation of reinforced concrete steel bars using piezoelectric sensors. Constr. Build. Mater. 2016, 105, 227-244. [CrossRef]

18. Dubov, A.A. A rapid method of inspecting welded joints utilizing the magnetic memory of metal. Weld. Int. 1997, 11, 410-413. [CrossRef] 
19. Dubov, A.A. Principle features of metal magnetic memory method and inspection tools as compared to known magnetic NDT methods. Can. Inst. NDE J. 2006, 27, 16-20.

20. Dubov, A.A. Diagnostics of equipment and construction strength with usage of magnetic memory. Insp. Diagn. 2001, 6, 19-29.

21. Jiang, S.T.; Li, W. Condensed Magnetic Physics, 1st ed.; China Science Publishing \& Media Ltd.: Beijing, China, 2003; pp. 1-16.

22. Zhong, W.D. Ferromagnetics (Volumen II), 1st ed.; China Science Publishing \& Media Ltd.: Beijing, China, 1987; pp. 3-9.

23. Wang, P.; Zhu, S.; Tian, G.Y.; Wang, H.; Wang, X. Stress Measurement Using Magnetic Barkhausen Noise and Metal Magnetic Memory Testing. In Proceedings of the 17th World Conference on Nondestructive Testing, Shanghai, China, 25-28 October 2008.

24. Doubov, A.A. Study of metal properties using the method of magnetic memory. Met. Sci. Heat Treat. 1997, 39, 401-405. [CrossRef]

25. Dubov, A.A.; Demin, E.A.; Milyaev, A.I.; Steklov, O.I. The experience of gas pipeline stress-strain state control with usage of the metal magnetic method as compared with conventional methods and stress control means. Weld. World 2002, 46, 29-33. [CrossRef]

26. Li, L.M.; Huang, S.L.; Wang, L.F.; Yang, H.Q.; Shi, K.R. Research on magnetic testing method of stress distribution. Trans. Nonferrous Mct. Soc. China 2002, 12, 388-391.

27. Huang, S.L.; Li, L.M.; Shi, K.R.; Wang, X.F. Magnetic field properties caused by stress concentration. J. Cent. S. Univ. Technol. 2004, 11, 23-26. [CrossRef]

28. Usarek, Z.; Chmielewski, M.; Piotrowski, L. Reduction of the velocity impact on the magnetic flux leakage signal. J. Nondestr. Eval. 2019, 38, 28. [CrossRef]

29. Ege, Y.; Coramik, M. A New Measurement system using magnetic flux leakage method in pipeline inspection. Measurement 2018, 123, 163-174. [CrossRef]

30. Kostin, V.N.; Filatenkov, D.Y.; Chekasina, Y.A.; Vasilenko, O.N.; Serbin, E.D. Features of excitation and detection of magnetoacoustic emission in ferromagnetic objects. Acoust. Phys. 2017, 63, 237-244. [CrossRef]

31. Schuster, S.; Dertinger, L.; Dapprich, D.; Gibmeier, J. Application of magnetic Barkhausen noise for residual stress analysis-consideration of the microstructure. Mater. Test. 2018, 60, 545-552. [CrossRef]

32. Zhang, H.; Liao, L.; Zhao, R.Q.; Zhou, J.T.; Yang, M.; Xia, R.C. The non-destructive test of steel corrosion in reinforced concrete bridge using a micro-magnetic sensor. Sensors 2016, 16, 1439. [CrossRef]

33. Dubov, A.A. Problems in estimating the remaining life of aging equipment. Therm. Eng. 2003, 11, 935-938.

34. Dubov, A.A. The method of metal magnetic memory-The new trend in engineering diagnostics. Weld. World 2005, 9, 314-319.

35. Qiu, J.; Zhang, H.; Zhou, J.; Ma, H.; Liao, L. Experimental analysis of the correlation between bending strength and SMFL of corroded RC beams. Constr. Build. Mater. 2019, 214, 594-605. [CrossRef]

36. Wang, Z.D.; Gu, Y.; Wang, Y.S. A review of three magnetic NDT technologies. J. Magn. Magn. Mater. 2012, 324, 382-388. [CrossRef]

37. Dong, S.Y.; Wang, D.; Xu, B.S.; Shi, C.L. Characterizing stress concentration by metal magnetic memory signal of $\mathrm{Hp}(x)$. Int. J. Appl. Electromagnet Mech. 2010, 33, 1219-1223. [CrossRef]

38. Wang, Z.D.; Yao, K.; Deng, B.; Ding, K.Q. Quantitative study of metal magnetic memory signals versus local stress concentration. NDT E Int. 2010, 43, 513-518. [CrossRef]

39. Chen, C.R.; Zeng, S.J.; Su, L.Y. Strain energy based method for metal magnetic memory effect of tensile tested structures. J. Nondestr. Eval. 2019, 38, 36. [CrossRef]

40. Yu, M.; Gou, R.B.; Zhang, C.Y.; Qiao, Y.H.; Si, Z.Y.; Xie, Z.S. Research on the relationship between metal magnetic memory signal distortion and yield strain under static tension test. Insight-Non-destr. Test. Condition Monit. 2014, 12, 669-675.

41. Pang, C.Y.; Zhou, J.T.; Zhao, R.Q.; Ma, H.; Zhou, Y. Research on internal force detection method of steel bar in elastic and yielding stage based on metal magnetic memory. Materials 2019, 12, 1167. [CrossRef]

42. Erber, T.; Guralnickz, S.A.; Desaiz, R.D.; Kwokx, W. Piezomagnetism and fatigue. J. Phys. D: Appl. Phys. 1997, 20, 2818-2836. [CrossRef] 
43. Bao, S.; Jin, W.L.; Huang, M.F.; Bai, Y. Piezomagnetic hysteresis as a non-destructive measure of the metal fatigue process. NDT E Int. 2010, 8, 706-712. [CrossRef]

44. Yi, X.R.; Tao, G.L.; Hu, Z.L. Effects of strengthening reinforced concrete chimneys with prestressed hoops. Eng. Mech. 2006, 23, 109-113.

(C) 2019 by the authors. Licensee MDPI, Basel, Switzerland. This article is an open access article distributed under the terms and conditions of the Creative Commons Attribution (CC BY) license (http://creativecommons.org/licenses/by/4.0/). 